\title{
Multidrug resistance protein 2 genetic polymorphism and colorectal cancer recurrence in patients receiving adjuvant FOLFOX-4 chemotherapy
}

\author{
MOJGAN MIRAKHORLI $^{1}$, SABARIAH ABDUL RAHMAN ${ }^{1,2}$, SYAHRILNIZAM ABDULLAH $^{3}$ \\ MASOUD VAKILI $^{4}$, REZA ROZAFZON ${ }^{5}$ and AHAD KHOSHZABAN ${ }^{5,6}$
}

\begin{abstract}
${ }^{1}$ Department of Pathology, Faculty of Medicine and Health Sciences, University Putra Malaysia, Serdang, Selangor 43400;
${ }^{2}$ Cluster of Medical Laboratory Sciences, Faculty of Medicine, Universiti of Teknologi MARA, Selayang Campus,

Batu Caves, Selangor $68100 ;{ }^{3}$ Medical Genetics Laboratory, Faculty of Medicine and Health Sciences, University Putra Malaysia, UPM Serdang, Selangor 43400, Malaysia; ${ }^{4}$ Department of Oncology, Hazrate Rasoul Akram Hospital, Tehran University of Medical Science; ${ }^{5}$ Stem Cell Preparation Unit, Eye Research Center, Farabi Eye Hospital; ${ }^{6}$ Department of Dental Biomaterial, Faculty of Dentistry, Tehran University of Medical Science, Tehran, Iran
\end{abstract}

Received June 30, 2012; Accepted October 25, 2012

DOI: $10.3892 / \mathrm{mmr} .2012 .1226$

\begin{abstract}
Multidrug resistance protein 2 (MRP2), encoded by the ATP-binding cassette C2 (ABCC2) gene, is an efflux pump located on the apical membrane of many polarized cells, which transports conjugate compounds by an ATP-dependent mechanism. The correlation of G1249A ABCC2 polymorphism with the development of colorectal cancer (CRC) and poor prognosis was evaluated in patients who were treated with fluorouracil/ leucovorin (FL) plus oxaliplatin (FOLFOX-4). A total of 50 paraffin-embedded tissue samples collected from CRC patients were analyzed to identify the polymorphism. Patients were in stage II/III and received postoperative FOLFOX-4 chemotherapy. As a control group, an equal number of unrelated healthy subjects were enrolled in the study. The polymorphism was genotyped by the polymerase chain reaction-restriction fragment length polymorphism (PCR-RFLP) method, and results were compared with clinicopathological markers, early relapse and survival rates. During the 12 months of follow-up, local and distant recurrences were observed in 15 (30\%) patients. No significant difference in the distribution of wild-type and polymorphic genotypes was observed between the patient and control groups and between the patients who experienced recurrence within 1 year and those who did not (all $\mathrm{P}>0.05$ ). In conclusion, the G1249A polymorphism is not associated with CRC risk and early recurrence. However, significant correlation was observed between G1249A polymorphism
\end{abstract}

Correspondence to: Professor Sabariah Abdul Rahman, Cluster of Medical Laboratory Sciences, Faculty of Medicine, Universiti of Teknologi MARA, Selayang Campus, Batu Caves, Selangor 68100, Malaysia

E-mail: sabariah@medic.upm.edu.my

Key words: G1249A, ABCC2/MRP1, colorectal cancer, recurrence, survival, oxaliplatin and the overall survival and disease-free survival of the patients.

\section{Introduction}

Adjuvant FOLFOX-4 chemotherapy following surgery is recommended as an effective therapy for patients with stage II and III colon cancer (1). Nevertheless, a high percentage of patients encounter recurrence as a primary cause of mortality, which is mainly associated with chemotherapeutic response (2-4). Over the past 10 years, combination chemotherapies have improved response rates and prolonged overall survival in colorectal cancer (CRC) patients (5). To date, oxaliplatin (a platinum drug) combined with fluorouracil (5FU)/leucovorin (FL) in the FOLFOX-4 regimen is frequently prescribed to treat $\mathrm{CRC}$.

The prediction of sensitivity or resistance to chemotherapy by analysis of genetic variations is of major interest in choosing the first-line chemotherapy most likely to be efficient. A single nucleotide polymorphism (SNP) is a point mutation that is observed in $1 \%$ of a general population. Genetic polymorphisms in the sequences of drug transporter genes have been found to affect the therapeutic response, toxicity and survival of cancer patients. Furthermore, SNPs may affect CRC susceptibility when exposed to exogenous and endogenous carcinogenesis.

The formation of glutathione conjugates is a well-known mechanism by which platinating agents inhibit tumor growth. It is also known to be one of the mechanisms involved in the resistance to oxaliplatin in CRC (6). Glutathione conjugates may be substrates of ATP-binding cassette (ABC) multidrug transporters. For example, glutathione detoxifies oxaliplatin by conjugation (7,8); the conjugate is eliminated by MRPs/ABCCs, either alone, as a co-substrate, or in its conjugated form (9). Intracellular glutathione content may be reduced by the high expression of MRPs/ABCCs (10). 


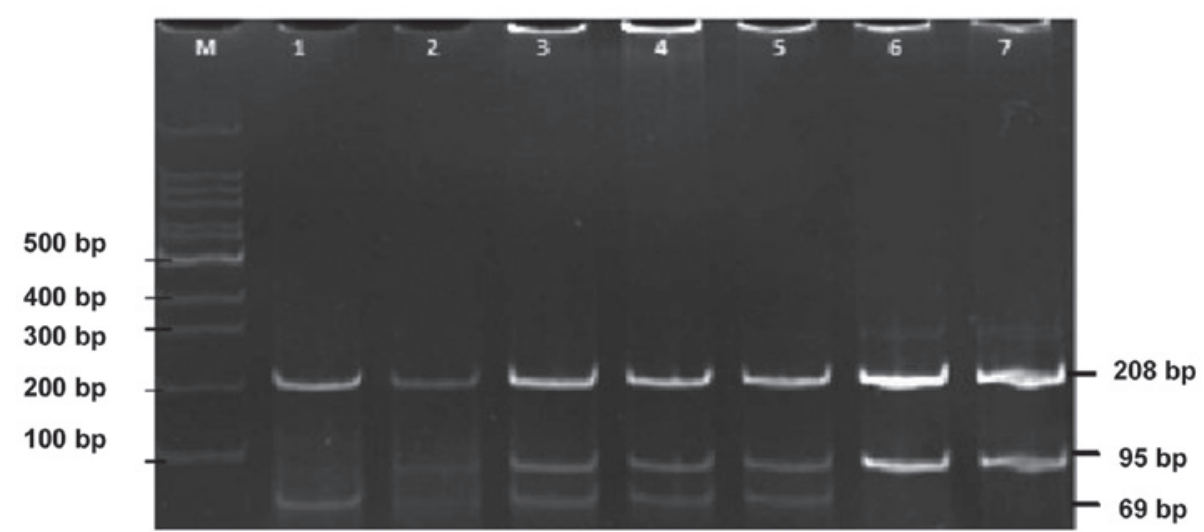

Figure 1. Electrophoresis pattern for MRP2 G1249A analyzed by a polymerase chain reaction-restriction fragment length polymorphism (PCR-RFLP)-based assay on 10\% polyacrylamide gel. M, marker: 100 bp; lane 1, homozygous AA: 208, 69 and 29 bp; lanes 2-5, homozygous GG: 208 and 95 bp; lanes 6 and 7 , heterozygous GA: 208, 95, 69 and 29 bp.

The human multidrug resistance protein 2 (MRP2/ABCC2/ cMOAT) gene is a member of the $\mathrm{ABC}$ transporter family that is located on chromosomal locus 10q24 and consists of 32 exons (11). MRP2 is presented at the apical membrane of polarized cells, including hepatocytes, renal proximal tubule epithelia and intestinal epithelia (12). It is the optimal pump to eliminate relatively hydrophilic compounds, including glucuronide, glutathione and sulfate conjugates (12). Among several MRP2 SNPs, the G1249A SNP is known to be associated with alterations in mRNA levels $(13,14)$. This MRP2 polymorphism results in an amino acid alteration from Val to Ile at position 417 , located in membrane spanning domain 2 of the protein. According to previous reports $(15,16)$, mutations in this domain of MRP2 may alter the specificity of its substrates but not its transporter activity. In the present study, we investigated the association of G1249A MRP2 polymorphism with the poor response to FOLFOX-4 chemotherapy, a platinum-base regimen, and the short-term survival of CRC patients.

\section{Materials and methods}

Patients and treatment. The subjects included 50 primary CRC patients (30 male, 20 female; median age 57 years), who received radical resection in Chamran Hospital and Hazrat Rasuol Akram Hospital, Tehran, Iran. Patients were eligible if they had undergone adjuvant FOLFOX-4 chemotherapy following the radical resection of histologically confirmed stage II (T2 and T3, N0, M0) or stage III (any T, N1 and 2, M0) CRC. Patients who had received preoperative chemotherapy and radiotherapy were excluded. A total of 50 unrelated healthy individuals who did not have a family history of cancer and were gender/age-matched with the patients were also enrolled in this study. The protocol of the study was approved by the Faculty of Medicine and Health Sciences Ethics Committee, University Putra Malaysia.

The patients received 12 cycles of a FOLFOX- 4 regimen which consisted a 2-week cycle of oxaliplatin $\left(85 \mathrm{mg} / \mathrm{m}^{2}\right)$ combined with leucovorin $\left(200 \mathrm{mg} / \mathrm{m}^{2}\right)$ on day 1 , bolus $5 \mathrm{FU}$ $\left(400 \mathrm{mg} / \mathrm{m}^{2}\right)$ and continuous infusion of $5 \mathrm{FU}\left(600 \mathrm{mg} / \mathrm{m}^{2}\right)$. Follow-up consisted of a carcinoembryonic antigen (CEA) test at 3-month intervals for 2 years and at 6-month intervals thereafter. Colonoscopy and CT scans were usually performed at 6-month intervals in the first 2 years and annually thereafter; however, these tests were mandatory following an elevated CEA level. Development of new recurrent or metastatic lesions following surgery was considered as a relapse. Local relapse was histopathologically/cytologically confirmed by the examination of specimens. Written informed patient consent was obtained from the patients.

DNA extraction and genotyping. Sections $(5-10 \mu \mathrm{m})$ of paraffin-embedded tissues $(\mathrm{n}=50)$ were cut with a microtome and placed in a $1.5-\mathrm{ml}$ microcentrifuge tube. To avoid intersample contamination, the blade was cleaned with xylene after cutting each paraffin block. DNA was isolated from the paraffin-embedded tissue samples using a QiAamp DNA FFPE tissue kit (Qiagen, Hilden, Germany) according to the manufacturer's instructions. DNA was also extracted from 50 blood samples of the unrelated healthy individuals using a kit (Qiagen).

Single nucleotide polymorphism was analyzed using a polymerase chain reaction-restriction fragment length polymorphism (PCR-RFLP) technique. The following PCR primers were used for genotyping: forward, 5'-GGGCAAAGAAGTGTGTG GAT-3'; and reverse, 5'-ACATCAGGTTCACTGTTTCTC CCA-3' (17). The PCR amplification conditions consisted of an initial denaturation step at $94^{\circ} \mathrm{C}$ for $3 \mathrm{~min}$, followed by 35 cycles of a denaturation step at $94^{\circ} \mathrm{C}$ for $20 \mathrm{sec}$, an annealing step at $56.4^{\circ} \mathrm{C}$ for $15 \mathrm{sec}$ and an extension step at $72^{\circ} \mathrm{C}$ for $20 \mathrm{sec}$ with a final extension step at $72^{\circ} \mathrm{C}$ for $5 \mathrm{~min}$. The PCR products were digested with 5 units $\mathrm{NcoI}$ at $37^{\circ} \mathrm{C}$ for $15 \mathrm{~h}$. The digested products were thereafter electrophoresed on a $10 \%$ acrylamide gel followed by ethidium bromide staining for genotype determination. The fragments obtained were 208 and $95 \mathrm{bp}$ for the wild-type genotype GG; 208, 69 and 26 bp for the mutant genotype AA; and 208, 95, 69 and 26 bp for the GA genotype (Fig. 1). The accuracy of PCR-RFLP was confirmed by direct sequencing using a Big-Dye Terminator Cycle Sequencing kit (Applied Biosystems, Foster City, CA, USA).

Data analysis. Data analysis was performed using the SPSS software V11.0 (SPSS, Inc., Chicago, IL, USA). The $\chi^{2}$ and 
Table I. Patients and tumor characteristics.

\begin{tabular}{|c|c|c|}
\hline Characteristics & Total cases & $(\%)$ \\
\hline \multicolumn{3}{|l|}{ Gender } \\
\hline Male & 30 & 60 \\
\hline Female & 20 & 40 \\
\hline \multicolumn{3}{|l|}{ Age (years) } \\
\hline$>50$ & 38 & 76 \\
\hline$<50$ & 12 & 24 \\
\hline \multicolumn{3}{|l|}{ Tumor size (cm) } \\
\hline$<5$ & 28 & 56 \\
\hline$>5$ & 22 & 44 \\
\hline \multicolumn{3}{|l|}{ Location } \\
\hline Colon & 35 & 70 \\
\hline Rectum & 15 & 30 \\
\hline \multicolumn{3}{|c|}{ Depth of tumor invasion } \\
\hline $\mathrm{T} 4$ & 3 & 6 \\
\hline $\mathrm{T} 3$ & 47 & 94 \\
\hline \multicolumn{3}{|c|}{ Lymph node metastasis } \\
\hline Negative & 22 & 44 \\
\hline Positive & 28 & 56 \\
\hline \multicolumn{3}{|l|}{ Stage } \\
\hline II & 22 & 44 \\
\hline III & 28 & 56 \\
\hline \multicolumn{3}{|l|}{ Histology ${ }^{a}$} \\
\hline $\mathrm{WD}+\mathrm{MD}$ & 48 & 96 \\
\hline PD & 2 & 4 \\
\hline
\end{tabular}

${ }^{a} \mathrm{WD}$, well-differentiated; MD, moderately differentiated; PD, poorly differentiated.

Fisher's exact tests were used to analyze the data. Any possible association between the development of the disease and the studied polymorphism was evaluated by calculating odds ratios (ORs) and 95\% confidence intervals (CIs) from contingency tables and using a two-sided Fisher's exact test. Survival analysis was performed using the Kaplan-Meier method and differences between the survival curves were evaluated with a log-rank test. Disease-free survival (DFS) was defined in months from the day of surgery to the first event of documented relapse or death. For those patients who did not relapse, data were recorded during the last follow-up. Overall survival (OS) was defined as the time from the date of coloctomy to death; data on survivors were censored at the last follow-up.

\section{Results}

A total of 50 patients diagnosed with stage II/III CRC (median age 60 years and range 17-77 years) were enrolled in the study. Of these, $60 \%$ were males and $52 \%$ were in UICC stage III. Other clinicopathological characteristics of the patients are listed in Table I. All patients were treated with 12 cycles of the FOLFOX-4 regimen for 6 months. The follow-up duration was 24-48 months. Fifteen cases (30\%) experienced an early
Table II. Genotypes and allele frequencies of the G1249A polymorphism in 50 colorectal cancer (CRC) patients and healthy controls.

\begin{tabular}{lccccccc}
\hline & \multicolumn{3}{c}{ Genotype N (\%) } & & \multicolumn{2}{c}{ Allele (\%) } \\
\cline { 2 - 3 } \cline { 7 - 8 } & GG & GA & AA & & G & A \\
\hline Patient & $16(32)$ & $28(56)$ & $6(12)$ & & 60 & 40 \\
Control & $22(44)$ & $23(46)$ & $5(10)$ & & 67 & 33 \\
P-value & 0.151 & 0.121 & 0.500 & & 0.189 & - \\
Odds ratio & 2.597 & 0.667 & 0.814 & & - & 0.739
\end{tabular}

Table III. Characteristics of patients according to G1249A genotypes.

\begin{tabular}{|c|c|c|c|}
\hline Characteristics & GG & GA or AA & P-value \\
\hline Gender & & & 0.247 \\
\hline Male $(n=30)$ & 8 & 22 & \\
\hline Female $(n=20)$ & 8 & 12 & \\
\hline Age (years) & & & 0.120 \\
\hline$>50(n=38)$ & 10 & 28 & \\
\hline$<50(\mathrm{n}=12)$ & 6 & 6 & \\
\hline Tumor size $(\mathrm{cm})$ & & & 0.120 \\
\hline$<5(\mathrm{n}=28)$ & 6 & 6 & \\
\hline$>5(n=22)$ & 10 & 28 & \\
\hline Location & & & 0.572 \\
\hline Colon $(n=35)$ & 11 & 24 & \\
\hline Rectum $(n=15)$ & 5 & 10 & \\
\hline Depth of tumor invasion & & & 0.237 \\
\hline T3 $(n=47)$ & 14 & 33 & \\
\hline $\mathrm{T} 4(\mathrm{n}=3)$ & 2 & 1 & \\
\hline Lymph node metastasis & & & 0.587 \\
\hline Negative $(n=22)$ & 8 & 14 & \\
\hline Positive $(n=28)$ & 8 & 20 & \\
\hline Stage & & & 0.587 \\
\hline II $(n=22)$ & 8 & 14 & \\
\hline III $(n=28)$ & 8 & 20 & \\
\hline Histology $^{a}$ & & & - \\
\hline $\mathrm{WD}+\mathrm{MD}(\mathrm{n}=48)$ & 14 & 34 & \\
\hline $\operatorname{PD}(n=2)$ & 2 & 0 & \\
\hline
\end{tabular}

${ }^{a} \mathrm{WD}$, well-differentiated; MD, moderately differentiated; PD, poorly differentiated.

relapse, either a local recurrence or distant metastasis, within 1 year of follow-up.

G1249A polymorphism and CRC risk. Table II presents OR estimates of CRC risk for each genotype of the MRP2 gene SNP. Of the 50 patients, the MDR2 wild-type genotype (1249GG) was observed in $32 \%$ of patients, whereas $56 \%$ were 


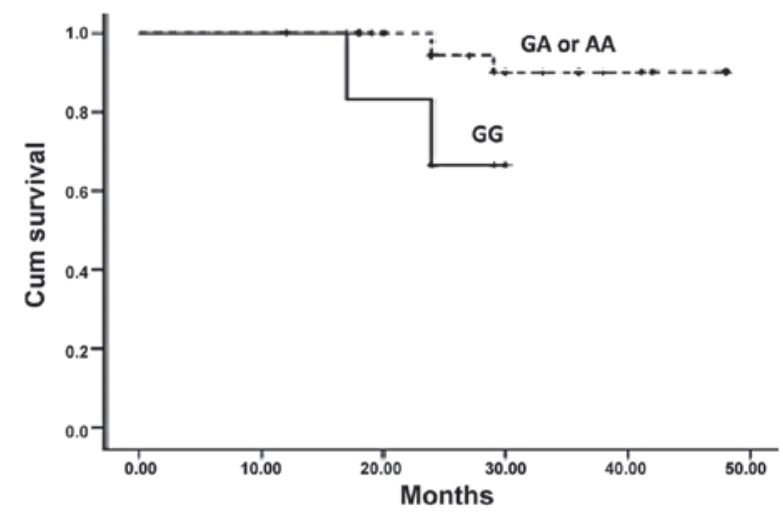

Figure 2. Effect of genotypes on the overall survival of patients with colorectal cancer (CRC). A significant association between G1249A polymorphism of MRP1 and overall survival was observed $(\mathrm{P}=0.043)$.

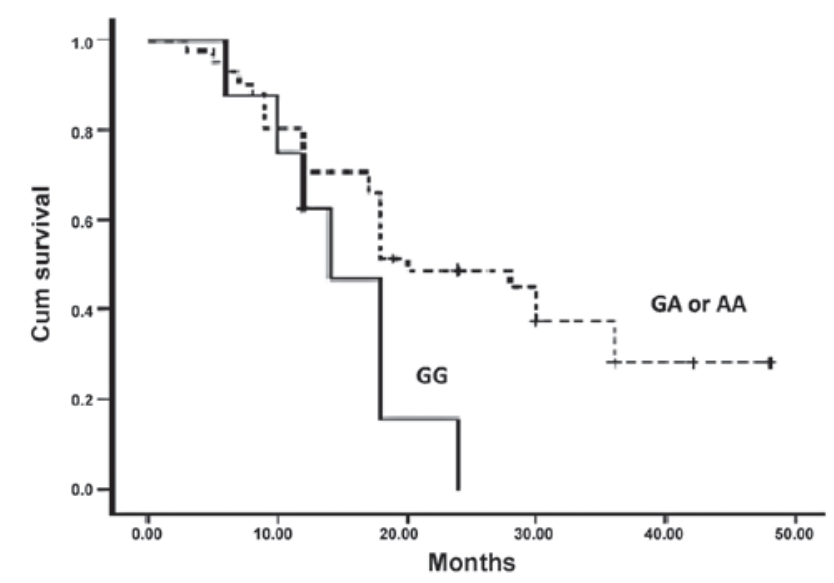

Figure 3. Effect of genotypes on the disease-free survival of patients with colorectal cancer (CRC). A significant association between G1249A polymorphism of MRP1 and disease-free survival was observed ( $\mathrm{P}=0.043)$.

heterozygous (1249GA) and 12\% were homozygous (1249AA) for the mutation. The 1249GG, 1249GA and 1249AA genotypes were found in 44, 46 and $10 \%$ of the controls, respectively. For the GG, AA and GA genotypes, no statistically significant association was identified between the SNP and CRC risk. In addition, no significant trend was identified in the number of alleles with respect to CRC occurrence.

Correlation between G1249A polymorphism and clinicopathological markers. No significant association was observed between the G1249A genotypes (GG, GA and AA) and the patient's characteristics, including age, gender, tumor location, tumor size, deep tumor invasion, disease stage and the presence or absence of lymph node metastasis ( $\mathrm{P}>0.05$; Table III).

Correlation between prognostic and clinicopathological markers. To investigate whether various clinicopathological data of the patients correlate with chemotherapy response, disease recurrence was evaluated according to the clinicopathological factors. None of those factors were identified to be associated with clinical response in the $\mathrm{CRC}$ patients $(\mathrm{P}>0.05)$. In addition, all factors were examined using the Kaplan-Meier method.
These parameters did not correlate with the OS or DFS of the patients (all $\mathrm{P}>0.05$ ), with the exception of deep tumor invasion. The correlation between deep tumor invasion and survival rates, although significant, was not suitable for evaluation, since the number of patients with T4 type tumors was very small.

Correlation between G1249A polymorphism and prognosis. To identify any possible correlation between the genotypes of the G1249A polymorphism and the survival rates and early recurrence, the patients were divided into the normal group (GG) and the mutation group (GA and AA). The polymorphic genotype of MRP2 was not different between the patients who responded and those who did not respond to the adjuvant FOLFOX-4 chemotherapy $(\mathrm{P}=0.468, \mathrm{P}>0.05)$. However, a significant association between G1249A genotypes and survival rates was observed when Kaplan-Meier survival curves were plotted (Figs. 2 and 3). Thus patients with a mutant allele A, either homozygous or heterozygous, of G1249A had higher DFS and OS than patients with wild-type allele $\mathrm{G}$ $(\mathrm{P}=0.045$ and 0.043$)$.

\section{Discussion}

Among platinum drugs, oxaliplatin is the favored drug for the therapy of CRC. Oxaliplatin combined with 5FU/LV (the FOLFOX-4 regimen) is currently used for the treatment of stage II/III CRC patients who have undergone complete resection of the primary tumor. Expression of ABC-transporter proteins, particularly MRP2, has been demonstrated to be associated with resistance to platinum-based anticancer drugs, including cisplatin $(18,19)$. Hence, variations in the MRP2 gene may be important for evaluating and/or predicting the response to platinum drugs. The role of MRP2 genetic polymorphisms on the response of CRC to platinum-based chemotherapy has not previously been reported. Among the polymorphisms of the MRP2 gene, G1249A has been found to be a common SNP that affects mRNA levels $(13,14)$. Owing to its possible effect on gene expression, we anticipated that this polymorphism of the MRP2 gene may affect the tumor response to adjuvant FOLFOX-4 chemotherapy in CRC. To examine our hypothesis, we investigated whether functional polymorphism of G1249A in the MRP2 gene affected CRC occurrence and/or correlated with early recurrence in patients treated with the FOLFOX-4 regimen. We selected a homogeneous population in stage II/III who had not received any preoperative treatment. The genotype frequencies of the MRP2 G1249A polymorphism did not change during malignancy and were identical in the tumor and the matched normal tissues in our selected population. Therefore, de novo mutation of the MRP2 gene, which may affect cancer development and drug response, does not appear to occur in CRC. In addition, we analyzed the genetic polymorphism in the DNA isolated from paraffin-embedded samples obtained from the patients' matched normal tissues and in the peripheral blood of the controls. A previous study has verified that the genotyping results of DNA isolated from tissue are equivalent to those of DNA isolated from blood (20). The frequency of the A allele for the G1249A polymorphism in the controls was 33\%, which is inconsistent with that reported in the literature (11). No association was observed in the present study between the SNP of 
the MRP2 gene in exon 10 and the development of CRC. By contrast, an association between this polymorphic genotype and the risk of primary colorectal adenocarcinoma has been previously reported in Japanese patients (21).

We have also analyzed this SNP to investigate its significance in relation to platinum-based chemotherapy. The genotype frequencies of G1249A were not different between the patients who relapsed within one year and those who did not. Our results indicate that the mutant allele in exon 10 was not related to the response to adjuvant FOLFOX-4 chemotherapy. Similar results were obtained by Sun et al (11) in Chinese advanced non-small cell lung cancer (NSCLC) patients who were treated with a platinum-based drug. By contrast, a significant correlation between G1249A and the response to chemotherapy was observed in advanced ovarian cancer by Xingsheng et al (22). In addition, in Kaplan-Meier curves, the adenocarcinoma patients with a GG genotype of G1249A demonstrated a significantly shorter overall survival and disease-free survival than patients with AA or GA genotypes. Thus, we demonstrated that G1249A MRP2 is a molecular predictive marker for the survival of patients with stage II/III CRC treated with adjuvant FOLFOX-4 therapy following curative resection.

In conclusion, the MRP2 G1249A polymorphism was not associated with the incidence of CRC in the Iranian population. In addition, this polymorphism did not affect the prognosis of the disease in our population. Although, the considered patient population was small in size, it was very homogeneous, and hence was suitable for prognostic evaluation.

\section{References}

1. Kuebler JP, Wieand HS, O'Connell MJ, et al: Oxaliplatin combined with weekly bolus fluorouracil and leucovorin as surgical adjuvant chemotherapy for stage II and III colon cancer: results from NSABP C-07. J Clin Oncol 25: 2198-2204, 2007.

2. Chen LT and Whang-Peng J: Current status of clinical studies for colorectal cancer in Taiwan. Clin Colorectal Cancer 4: 196-203, 2004.

3. de Gramont AD, Tournigand C, André T, Larsen AK and Louvet C: Adjuvant therapy for stage II and III colorectal cancer. Semin Oncol 34 (Suppl 1): S37-S40, 2007.

4. Schippinger W, Samonigg H, Schaberl-Moser R, et al: A prospective randomised phase III trial of adjuvant chemotherapy with 5-fluorouracil and leucovorin in patients with stage II colon cancer. Br J Cancer 97: 1021-1027, 2007.

5. Gonzalez-Haba E, García MI, Cortejoso L, et al: ABCB1 gene polymorphisms are associated with adverse reactions in fluoropyrimidine-treated colorectal cancer patients. Pharmacogenomics 11: 1715-1723, 2010.

6. Casado E, De Castro J, Belda-Iniesta C, et al: Molecular markers in colorectal cancer: genetic bases for a customised treatment. Clin Transl Oncol 9: 549-554, 2007.
7. Perez RP, Hamilton TC and Ozols RF: Resistance to alkylating agents and cisplatin: insights from ovarian carcinoma model systems. Pharmacol Ther 48: 19-27, 1990.

8. El-akawi Z, Abu-hadid M, Perez R, et al: Altered glutathione metabolism in oxaliplatin resistant ovarian carcinoma cells. Cancer Lett 105: 5-14, 1996.

9. Theile D, Grebhardt S, Haefeli WE and Weiss J: Involvement of drug transporters in the synergistic action of FOLFOX combination chemotherapy. Biochem Pharmacol 78: 1366-1373, 2009.

10. Ballatori N, Hammond CL, Cunningham JB, Krance SM and Marchan R: Molecular mechanisms of reduced glutathione transport: role of the MRP/CFTR/ABCC and OATP/SLC21A families of membrane proteins. Toxicol Appl Pharmacol 204: 238-255, 2005.

11. Sun N, Sun X, Chen B, et al: MRP2 and GSTP1 polymorphisms and chemotherapy response in advanced non-small cell lung cancer. Cancer Chemother Pharmacol 65: 437-446, 2010.

12. Nies AT and Keppler D: The apical conjugate efflux pump ABCC2 (MRP2). Pflugers Arch 453: 643-659, 2007.

13. Meyer zu Schwabedissen HE, Jedlitschky G, Gratz M, et al: Variable expression of MRP2 (ABCC2) in human placenta: influence of gestational age and cellular differentiation. Drug Metab Dispos 33: 896-904, 2005.

14. Kroetz DL, Liu W, Nguyen TD, et al: $1249 \mathrm{G}>\mathrm{A}$ polymorphism of $A B C C 2$ (MRP2) is associated with altered gene expression in human liver. J Clin Oncol 24 (Suppl): 13072, 2006.

15. Toh S, Wada M, Uchiumi T, et al: Genomic structure of the canalicular multispecific organic anion-transporter gene (MRP2/cMOAT) and mutations in the ATP-binding-cassette region in Dubin-Johnson syndrome. Am J Hum Genet 64: 739-746, 1999.

16. Choi JH, Ahn BM, Yi J, et al: MRP2 haplotypes confer differential susceptibility to toxic liver injury. Pharmacogenet Genomics 17: 403-415, 2007.

17. Naesens M, Kuypers DR, Verbeke K and Vanrenterghem Y: Multidrug resistance protein 2 genetic polymorphisms influence mycophenolic acid exposure in renal allograft recipients. Transplantation 82: 1074-1084, 2006.

18. Liedert B, Materna V, Schadendorf D, Thomale $\mathrm{J}$ and Lage H: Overexpression of cMOAT (MRP2/ABCC2) is associated with decreased formation of platinum-DNA adducts and decreased G2-arrest in melanoma cells resistant to cisplatin. J Invest Dermatol 121: 172-176, 2003.

19. Shen DW, Goldenberg S, Pastan I and Gottesman MM: Decreased accumulation of [14C]carboplatin in human cisplatin-resistant cells results from reduced energy-dependent uptake. J Cell Physiol 183: 108-116, 2000.

20. Petrova DT, Nedeva P, Maslyankov S, et al: No association between MDR1 (ABCB1) $2677 \mathrm{G}>\mathrm{T}$ and $3435 \mathrm{C}>\mathrm{T}$ polymorphism and sporadic colorectal cancer among Bulgarian patients. J Cancer Res Clin Oncol 134: 317-322, 2008.

21. Nishioka C, Sakaeda T, Nakamura T, et al: MDR1, MRP1 and MRP2 genotypes and in vitro chemosensitivity in Japanese patients with colorectal adenocarcinomas. Kobe J Med Sci 50: 181-188, 2004

22. Xingsheng Y, Sekine M, Kurata H, et al: Single nucleotide polymorphisms in MRP2 gene and their significance on chemosensitivity of advanced ovarian cancer. J Pract Obstet Gynecol 20: 153-155, 2004. 